Revista Educação e Políticas em Debate - v. 7, n.1, p. 191 - 207 - jan./abr. 2018 - ISSN 2238-8346

\title{
O Déclassement visto a partir das propostas teóricas de G. Friedman Y P. Naville
}

\author{
The Déclassement seen from the theoretical proposals of G. Friedman Y. P. Naville \\ Le Déclassement vu des propositions théoriques de G. Friedman Y. P. Naville
}

Tania Aillón Gónez ${ }^{1}$

Universidad Mayor de San Simón

\begin{abstract}
Resumo: Este artigo analisa o alcance explicativo dos postulados teóricos e epistemológicos de G. Friedman e P. Naville, sobre um fenômeno que se disseminou nos mercados de trabalho contemporâneos, o déclassement. Com essa reflexão nos colocamos dentro da problemática da relação entre formação e emprego, ao mesmo tempo em que atualizamos um dos debates clássicos da Sociologia do Trabalho e da Sociologia da Educação, o debate sobre a qualificação, como reconhecimento da formação profissional. Para esta finalidade, recorremos a informações coletadas por pesquisas realizadas em diferentes latitudes, a fim de medir a amplitude e a importância de nosso objeto de reflexão, o déclassement.
\end{abstract}

Palavras-chave: Déclassement. Qualificação. Mercado de trabalho. Formação profissional. Emprego.

Abstract: This article analyzes the explanatory scope of the theoretical and epistemological postulates of G. Friedman and P. Naville on a phenomenon that has spread in the contemporary job markets: the déclassement. With this reflection we place ourselves within the problematic of the relationship between education and employment, while at the same time updating one of the classic debates of Sociology of Work and Sociology of Education, the debate about qualification as recognition of professional education. For this purpose, we resorted to information collected by researches carried out in different latitudes, in order to measure the breadth and importance of our object of reflection, the déclassement.

Keywords: Déclassement. Qualification. Job market. Professional qualification. Job.

Resumé: Cet article analyse la portée explicative des postulats théoriques et épistémologiques de $g$. Friedman et $P$. Naville sur un phénomène qui s'est disséminé sur les marchés du travail contemporains: le déclassement. Cette réflexion nous place au cour de la problématique de la relation entre formation et emploi en même temps qu'elle met à jour un des débats classiques de la Sociologie du Travail et de la Sociologie de l'Éducation, celui de la qualification en tant que reconnaissance de la formation professionnelle. Pour ce faire, nous avons recours à des informations provenant de recherches réalisées sous différentes latitudes afin de mesurer l'ampleur et l'importance de notre objet de réflexion: le déclassement.

\footnotetext{
${ }^{1}$ Socióloga economista, miembro fundador del Grupo de Estudios del Trabajo Llank'aymanta y docente investigadora del Instituto de Estudios Sociales y Económicos de la Universidad Mayor de San Dimón. E-mail: ledaillon@hotmail.com.
} 
Mots-clés: Déclassement. Qualification. Marché du travail. Formation professionnelle. Emploi.

\section{Planteamiento de la cuestion}

La dinámica del capitalismo contemporáneo, se caracteriza por profundas trasformaciones en la configuración de los mercados de trabajo, resultado del retroceso político de la clase obrera, expresado en el avance de reformas patronales tendientes a flexibilizar los mercados de trabajo, a medida que se extiende la automatización en los procesos productivos. Tanto la flexibilización, como la automatización han contribuido a la expansión de un ejército industrial de reserva, que, en periodos de crisis económica, aumenta su caudal de desocupados. Ante la perspectiva de enfrentar las consecuencias de un paro prolongado, muchos trabajadores se ven obligados a aceptar puestos vacantes que no corresponden con su nivel de formación (DI PAULA Y MOULLIET, 2009, entre otros autores), en tanto que el patrón está en condiciones de seleccionar candidatos e incluso de escoger a los más calificados.

Esta situación, se ha visto afianzada por la masificación de la educación, que cambió los sistemas educativos y contribuyó a la presencia de importantes contingentes de fuerza de trabajo calificada en los mercados de trabajo. El valor de diplomas idénticos, situados; aproximadamente, en el mismo rango de jerarquía, tiende a bajar de una generación a otra, por el proceso de masificación (MONCEL, 2012;). Numerosos observadores se interrogan sobre la adecuación real de la oferta de trabajo educada, a la demanda del mercado de trabajo, a medida que se constata una incongruencia, entre las competencias adquiridas en el sistema educativo y los requerimientos en los puestos de trabajo, particularmente, en el caso de las generaciones jóvenes, al punto que algunos autores, hablan de un proceso de desvalorización de los títulos escolares, debido a su amplia difusión (Plassard y Thi Thanh Nhu Tran, 2009; Laurance, 2005). 
En este contexto, se extendió en las últimas décadas, en los mercados de trabajo, un fenómeno que se conoce como déclassement profesional, surcalitication y/o sobrecalificación², que se produce, cuando las personas resultan tener competencias superiores en relación al puesto de trabajo que ocupan, estableciéndose así, una diferencia, entre una trayectoria laboral esperada y una trayectoria real. Estar en situación de déclassement, significa; entonces, poseer un nivel de formación superior al requerido por el empleo ocupado (un licenciado en derecho convertido en un animador sociocultural) (ECKERT, 2014)3. Como el “declassement" se sitúa dentro la problemática de la relación formación-empleo, en este artículo, constituye nuestra puerta de entrada, para reflexionar los alcances heurísticos, que tienen las propuestas teóricas de George Friedman y Pierre Naville, para explicarlo y comprenderlo, reactualizando una vez más, uno de los debates clásicos de la Sociología del Trabajo, el de la cualificación, en tanto reconocimiento social y económico a la formación profesional.

\section{Las cualificaciones desde la mirada de G. Friedman Y P. Naville}

El estudio de las cualificaciones profesionales, aparece como un problema de reflexión en la Sociología del Trabajo y en la Sociología de la Educación, debido al crecimiento de la parcelación de tareas, que acompañó el desarrollo de la mecanización en la industria; sobre todo, después de la segunda guerra mundial. En una época marcada por la introducción de la cadena en la gran industria y la descomposición en los oficios considerados, hasta entonces, tradicionales, uno de los debates constitutivos del nacimiento de la Sociología del Trabajo es el que se desarrolló alrededor de la cualificación del trabajo, distinguiendo la concepción denominada "sustancialista" de G. Friedmann de la visión identificada como "relativista" de P. Naville (ambos, reconocidos fundadores de la Sociología del Trabajo) (BUSCATTO, 2006).

\footnotetext{
${ }^{2}$ En este artículo, utilizaremos el concepto francés de déclassement, porque tiene una connotación, que no encontramos en los otros conceptos (sobrecalificacion o surcalification), una connotación dinámica, de desplazamiento a la baja en términos jerárquicos profesionales y sociales.

${ }^{3}$ Dentro de un periodo de crisis o a la salida de la crisis, cuando la diferencia entre formación y puesto ocupado se profundiza, emerge una concepción del declassement, que no se apoya en la inflexión de grupos sociales trayectorias familiares, sino sobre la comparación de un título detentado por un individuo y su inserción dentro del proceso productivo, es decir, se centra en la desviación, entre la promesa del título y el empleo efectivo (Eckert, $2014)$.
} 


\subsection{El contenido de las tareas como medida de las cualificaciones en Friedman}

No encontramos en G. Friedman un concepto explícito sobre cualificación, aunque ella esté en el centro de su problemática de investigación. En una de sus obras como: "Los problemas humanos del maquinismo" (1956), se encuentra una concepción implícita de la cualificación, que nos remite a la habilidad profesional, a los conocimientos profesionales o técnicos, a la inteligencia profesional, al aprendizaje individual durante varios años ${ }^{4}$, al manejo de la maquinaria, a la iniciativa en los métodos de trabajo, en la selección de herramientas o en la rapidez de las operaciones. El obrero calificado sobre su máquina es un profesional, el conoce el material, su estructura, su cualidad, el posee, en los oficios, la habilidad manual; delicada; precisa; es decir, que, en Friedman, la cualificación será igualada a la técnica empleada., por lo que su definición retoma los rasgos artesanales del trabajo, la cualificación como la expresión de una personalidad, de rasgos naturales, considerados eminentemente subjetivos (GARCÍA LÓPEZ, 2006).

En esta medida, muchos autores definen la concepción de G. Friedmann sobre las cualificaciones, como "sustancialista” (BUSCATTO, 2006), porque se centra en el contenido de las tareas, a través de una observación minuciosa de la habilidad profesional del oficio del obrero calificado (FRIEDMAN, 1956). De esta forma, G. Friedman se preguntará, frente a las conclusiones de Elton Mayo, acerca del enriquecimiento de las tareas ${ }^{5}$, si: ¿la deseada «recomposición de puestos» está acompañada necesariamente de una recualificación? ¿O por el contrario de una descualificación del trabajo obrero?, ¿cuáles son las nuevas necesidades de formación? ¿Se trata de un enriquecimiento o de un empobrecimiento de saberes necesarios? ¿Las «nuevas cualificaciones observadas en la industria y los servicios son realmente cualificaciones», susceptibles de transformarse en nuevos oficios? ¿O se trata; más bien, de saberes parcelados, ¿banales y sin un valor real en los mercados de trabajo?

\footnotetext{
${ }^{4}$ Evidentemente, existe una referencia al tiempo de formación como un factor importante de la cualificación en G. Friedman; sin embargo, como señala Stroobants (1993), tanto para G. Friedman como para Reynaud, en todo aprendizaje intervendrán tiempos analíticamente diferentes: el acostumbramiento, la iniciación y el dominio de los gajes del oficio. Tiempos que; además, variaran según el grado de organización del aprendizaje. Ante la inexistencia; entonces, de un denominador común para medir la cualificación, estos autores propusieron aprehenderla, a partir del trabajo mismo (GARCÍA LÓPEZ, 2006).

${ }^{5}$ Son famosos; en este sentido, los estudios realizados por Elton Mayo en la Hawthorne, en Estados Unidos, acerca de los efectos de las condiciones físicas de trabajo, sobre la productividad y la motivación de los obreros y el posible enriquecimiento en sus tareas, para estos fines. Estudios con los que se consolida el surgimiento de la denominada Escuela de las Relaciones Humanas.
} 
Estas preguntas definen una forma de aproximación al objeto de estudio y las características de los útiles de recojo y análisis de la información, los mismos que deben apuntar a captar las diferentes etapas del proceso de trabajo humano, para observar, la habilidad profesional del trabajador, con aplicación de un conjunto de instrumentos de medida o de comparación, para apreciar, por ejemplo, si existe una degradación de la cualificación en el taylorismo, en relación a las etapas anteriores. Con esta práctica investigativa, G. Friedman se consagra; sobre todo, a la observación directa de las situaciones de trabajo, por lo que algunos autores se referirán a él, como un investigador que restituyó la investigación empírica en Francia y que, como pionero de la investigación sociológica en el campo del trabajo, tuvo el mérito de crear los principales materiales descriptivos sobre los cuales trabajaran quienes continuaron sus investigaciones ${ }^{6}$. En este sentido, sí la cualificación es asimilada a la técnica empleada por el trabajador en su puesto de trabajo, la relación formación-empleo aparece como directa, propia de una concepción no mercantil del vínculo entre el trabajador y su trabajo $\left(\right.$ GARCÍA LÓPEZ, 2006) ${ }^{7}$.

\subsection{La cualificación como proceso históricamente condicionado en P.Naville}

P. Naville, parte de una perspectiva opuesta a la de G Friedman, para definir la cualificación; en este autor, la tarea no adquiere una significación, propiamente sociológica si es analizada; exclusivamente, desde la relación individual y directa entre la persona, el material, la herramienta y el producto. El taller y sus situaciones de trabajo no son, sino, un momento en una actividad productiva global que se extiende a diversas cadenas de empresas y, poco a poco, implica a ramas industriales enteras. En este sentido, no es el hecho técnico en sí y por sí mismo, el que provoca, directamente, efectos sociales, sino ese mismo hecho técnico, en tanto que expresión de determinadas relaciones sociales y económicas, históricamente específicas.

\footnotetext{
${ }^{6}$ Entre sus seguidores sobresale Alain Touraine, cuyas investigaciones contribuyeron a hacer avanzar el conocimiento en la Sociología del Trabajo y; en este sentido, las posiciones planteadas por el mismo Friedman.

${ }^{7}$ Esta concepción tuvo mucha influencia en el desarrollo, no solo de la investigación académica; sino también, de la forma en la que se encaran las políticas educativas en países como Francia. El CEREQ (una institución al servicio de los actores relacionados con la formación y el empleo en Francia, combina la producción de estadísticas, investigaciones y estudios con el acompañamiento de acciones concretas, como formular diagnósticos y pronósticos destinados a orientar las políticas de formación a escala regional, nacional e internacional) concentró su interés, en la búsqueda de la adecuación de la formación al empleo, para dar cuenta de la jerarquía del trabajo, porque concebía que la tarea consistía, en la acción directa sobre la oferta de formación (Tanguy, 2008).
} 
Se trata; entonces, de situar el hecho técnico como resultado de procesos más bastos, como los cambios cualitativos que provocó la automatización en la estructura de la fuerza de trabajo (creciente movilidad de la fuerza de trabajo dentro del aparato productivo, y su mayor polivalencia funcional en empresas y sectores), al reforzar la separación del trabajador, respecto de su puesto de trabajo( separación característica de las relaciones sociales de producción capitalista), lo que lleva con mayor razón a interrogarse, acerca de la relación; aparentemente determinante, de las operaciones técnicas particulares(actividades directas de trabajo) sobre los estatutos laborales de los trabajadores (GARCÍA LÓPEZ, 2006). La cualificación, situada históricamente, supone; en la época contemporánea, considerarla dentro de los cambios que en la estructura de la fuerza de trabajo, demandan los procesos automatizados; es decir, la creciente versatilidad de conocimientos y habilidades movilizadas en torno a nuevas secuencias productivas que dificultan una definición estrictamente técnica de la cualificación, porque no es posible una determinación operacional del puesto fijado a la estructura funcional de un determinado aparato productivo, al mismo tiempo que, los nuevos puntos de aplicación de la fuerza de trabajo, demandan del trabajador predisposiciones como su responsabilidad, su voluntad de cooperar, su adaptabilidad a la flexibilidad funcional, etc.(GARCÍA LÓPEZ, 2006).

De esta forma, para P. Naville no existe un medio directo y objetivo de cualificar un conjunto de puestos de trabajo por razones puramente técnicas, la única medida que tiene un sentido es la medida por el tiempo de formación, tal y como la sociedad lo exige en sus diferentes y variables niveles que ella paga por utilizar (NAVILLE, 1978). Lo que cualifica; en este caso, no es un tiempo de trabajo individual, analíticamente diferente (como sostiene G. Friedman), sino un tiempo socialmente necesario para producir un determinado grado de formación. La cualificación es para Naville, el resultado de una comparación social del tiempo de formación, entre las diversas fuerzas de trabajo, no consideradas por sus cualidades inmediatas(la habilidad profesional, los conocimientos profesionales o técnicos, la inteligencia profesional, el aprendizaje individual durante varios años), sino por una medida común, una determinada duración en el tiempo (GARCÍA LÓPEZ, 2006) de trabajo socialmente necesario,

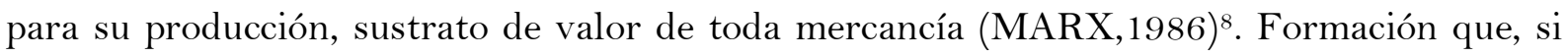
bien resulta de aprendizajes variados, solo se hace comparable, en función, de algo común a

\footnotetext{
${ }^{8} \mathrm{El}$ "tiempo socialmente necesario" no es una causa o variable independiente "natural" sino un producto social complejo en permanente movimiento: "El tiempo de trabajo socialmente necesario (...) es (...) una relación social compleja entre los hombres, sus actividades y los medios de producción, relación reflejada, invertida y coagulada en (...) representaciones sociales [y no] una especie de medida natural (...)” (Vincent, 1987, p. 106 y 107).
} 
todos estos diversos aprendizajes, el tiempo de formación, elemento general y socialmente valorizado. Solo en la medida en que las capacidades laborales sean juzgadas en relación a la unidad de medida común que impone el "tiempo de aprendizaje", entendido en un sentido amplio (duración de los estudios, costo de aprendizaje, capacidades adquiridas, antigüedad), ellas pueden llegar a cualificar el trabajo (NAVILLE, 1979). De estas capacidades y habilidades; solo una parte servirá para cualificar, es decir, para clasificar y jerarquizar las actuaciones de unos trabajadores, respecto de otros, en los espacios productivos. Esta distancia entre las competencias adquiridas en la formación y las reconocidas efectivamente, por los empresarios, (las “cualificaciones" "cualificantes"), se gestionan desde los procesos que tratan de sacar ventaja, tanto del mayor o menor nivel de institucionalización, como del mayor o menor nivel de socialización de la formación profesional (GARCÍA LÓPEZ, 2006).

En consecuencia, para P. Naville, la cualificación se descubre en el mercado de trabajo, como escasez social relativa a unas y otras capacidades de trabajo. La relación entre trabajo cualificado y trabajo no cualificado, así como las relaciones entre los distintos niveles de trabajo cualificado(toda característica de la fuerza de trabajo que se obtenga mediante una educación común empezara a formar parte del trabajo no cualificado), se encuentra en permanente movimiento; de ahí que la relación, entre la duración del aprendizaje, característica de una fuerza de trabajo y su remuneración no está establecida de una vez y para siempre, sino que resulta sensible a las condiciones del mercado de trabajo, a las transformaciones técnicas, socioeconómicas y culturales de las diferentes sociedades.

$\mathrm{El}$ análisis de las cualificaciones del trabajo no puede ser encerrado ni en el puesto de trabajo ni en la empresa, ni en la formación del título educativo, sino que debe extenderse hasta el movimiento ligado con las relaciones reciprocas que distribuyen las capacidades individuales entre las diferentes posiciones y modifican, sin cesar, sus posiciones y contenidos (NAVILLE, 1979). De esta concepción de la cualificación, se deduce que la relación entre formación y empleo, a la cual media, no es inmediata, porque ella misma es un proceso en continua transformación, una relación reconstruida de forma permanente, fruto de luchas y negociaciones entre patrones y trabajadores, bajo determinadas condiciones historicas ${ }^{9}$.

\footnotetext{
${ }^{9}$ Vale la pena precisar, que esta es una de las perspectivas epistemológicas y teóricas de la cualificación y de la relación formación -empleo, que en mucho menor grado, que la denominada "sustancialista", ha sido aplicada en las investigaciones en Sociología del Trabajo; principalmente, si nos referimos a la Sociología del Trabajo francesa.
} 


\section{Las teorías de G. Friedman Y P. Naville a la luz de la referencia empírica}

Para comprender los procesos contemporáneos de déclassement en los mercados de trabajo, utilizaremos información obtenida en distintas latitudes y con diferentes métodos de medición ${ }^{10}$, para valorarla, en relación a las perspectivas teóricas de G. Friedman y P. Naville, expuestas en el acápite anterior.

\subsection{El declassement: un indicador de la persistente separación entre formación y empleo}

La perspectiva de G. Friedman, que asimila la cualificación a la técnica empleada por el trabajador en su puesto de trabajo, donde la relación formación-empleo aparece como directa, tuvo mucha influencia en los espacios institucionales dedicados, no solo a los estudios en los mercados de trabajo; sino también, al diseño de políticas educativas que respondan a los requerimientos del aparato productivo, esto condujo a que la preocupación por el empleo, se centrara en la adecuación de la formación( la adquisición de competencias requeridas por el ejercicio del trabajo), como la dimensión estratégica; que no solo facilitaría el acceso a empleos de mejor calidad, sino también, garantizaría las ganancias de rendimiento y de productividad de las empresas. Se trata de una visión que perdura, en la medida en que gran parte de los estudios sobre el tema; en la actualidad, hacen aparecer a la formación como la condición fundamental de acceso al empleo, ocultando las relaciones sociales que presiden a esta relación entre títulos y espacios laborales (Tanguy, 2008). Sin embargo, esta visión instrumentalista, se puso a prueba cuando se fue identificando, en los mercados de trabajo, la discordancia entre la formación y la calidad del empleo; es decir, la aparición, cada vez más frecuente, de situaciones de déclassement profesional. Una muestra de que la contratación de personal, para las empresas, consideraba otros elementos, que iban más allá de la formación, hecho que ponía en cuestión una relación necesaria y directa entre formación y empleo (TANGUY, 2008).

En Europa, la preocupación por la incongruencia entre la formación y el empleo, motivó la realización de varios estudios centrados en los mercados de trabajo de los jóvenes

\footnotetext{
10 Para identificar el déclassement profesional existen al menos, tres métodos utilizados por los investigadores: uno de carácter normativo, que se basa en e l contenido de una formación necesaria que es a priori necesaria para ejercer un puesto. En este caso, intervienen expertos en educación o en mercados de trabajo que establecen estas correspondencias. Otro método de identificación y medida es el estadístico que define una norma en base análisis estadísticos, tomando en cuenta las situaciones más frecuentes, en este caso, habría déclassement, cuando la relación formación empleo se separa de esta norma establecida estadísticamente. Una tercera forma de identificación del "declassement" es de carácter subjetivo y se basa en captar la percepción que tienen las personas acerca de su empleo; en este caso, la norma es establecida por la persona interrogada (Giret et al., 2006).
} 
con diplomas superiores, donde el déclassement se hizo más notorio. En España, organismos internacionales como la Organización para la Cooperación y el Desarrollo Económicos (OCDE) han situado a este país, con el nivel más alto de déclassement entre sus miembros, con un nivel superior al doble de la media. Según otros estudios, tanto a nivel general (GARCÍAMONTALVO Y SORO, 2003) como entre los universitarios, en particular (GARCÍAMONTALVO, 2009), certificaron que el fenómeno del déclassement está muy extendido en el mercado laboral español. Estudios en Gran Bretaña (DOLTON Y VIGNOLES, 2000) y Francia comprueban que son los jóvenes con diplomas superiores los que en mayor grado que los adultos, ocupan puestos de trabajo por debajo de su nivel de formación.

En Francia, diversas estadísticas evidencian la presencia del déclassement profesional y salarial entre diplomados de la enseñanza superior. Guironnet (2009) en base a una encuesta de empleo, encontró una incongruencia entre diploma y remuneración, que la atribuyó al desarrollo de un déclassement estructural, para los diplomas de enseñanza superior en el periodo 1982-2002, que pasó de 2.6 en 1982 a 10\% en 2002. Cifras más recientes muestran que tres años después del fin de sus estudios, del 20 al 28\% de los jóvenes en 2010, ocupaban un puesto de trabajo que puede ser asimilado a una situación de déclassement (LEMISTRE, 2014).

Fuera de los resultados obtenidos, mediante el método normativo y/o estadístico, se encuentra el déclassement identificado, desde la vivencia de los implicados, a través de la opinión de las personas sobre la relación que encuentran, entre las competencias adquiridas y las requeridas en el empleo que desempeñan. Para generaciones de jóvenes, anteriores al 2007, entre 1998 y el año 2004, en Francia, numerosos estudiantes de master, que ocupan empleos considerados intermedios, consideraban que estaban empleados por debajo de su nivel de competencias (LEMISTRE, 2014).

Encontramos que el déclassemnt no solo afecta a los diplomas superiores, sino también, a los niveles más modestos de trabajadores, uno de estos casos, se encuentra en estudios como el de Guibert, Lazuech y Troger (2013), sobre el proceso de déclassement de un oficio, entre las mucamas de hoteles de lujo en Francia. En este caso, el déclassemet está inducido por los efectos de las nuevas tecnologías de racionalización, aplicadas por los managers (homogenización, formalización, de los oficios), que vuelven secundarias ciertas competencias profesionales, que constituyen la parte "noble" del oficio (el trato sofisticado y atento con una clientela de elite), convirtiendo a estas mucamas, de más en más, en simples técnicas de limpieza, sin autonomía, ni margen de maniobra. Se trata de un proceso de déclassement, que se traduce en la posibilidad, para los patrones, de introducir en este mercado de trabajo, a jóvenes que sin tener las 
competencias profesionales de las antiguas mucamas, se ajustan a los procesos de homogenización y formalización del oficio, acompañados de salarios comparativamente más bajos.

Fuera de la órbita europea, en A. Latina, estudios de la CEPAL (2006) identifican una marcada correlación relativa, entre el peso de los sectores de baja productividad y el nivel educativo de los jóvenes. En el contexto de un bajo dinamismo de las economías de la región, al mismo tiempo que se produce un aumento de nivel educativo de los jóvenes que entran al mercado de trabajo, se constata que un número creciente de estos jóvenes no encuentran un empleo acorde con su formación (CEPAL, 2006). En espacios más reducidos, en los mercados internos de trabajo de las empresas chinas, en Bolivia (caso que, dicho sea de paso, puede considerarse como una ilustración de proyectos de construcción, ejecutados por empresas chinas en otros países de la región), encontramos la práctica del déclassement bastante generalizada, como estrategia de reducción de costos laborales (Castro: 2018). Para esto, según testimonios obreros, los managers reducen el número necesario de puestos de trabajo con categoría de maestro constructor y amplían el número de puestos con categoría de ayudante; por lo que los obreros con categoría de maestros, se ven obligados a ocupar los puestos de ayudante, y, dentro de la dinámica de la obra de construcción, presionados por el ritmo de trabajo y los tiempos de entrega, terminan realizando las tareas de un maestro constructor.

La práctica del déclassement, la encontramos, también, entre trabajadoras del sector público en Bolivia. Se trata de licenciadas en ciencias jurídicas, contratadas por el Estado como “consultoras" bajo la modalidad de "venta de Servicios", a las que, en la práctica, se les hace realizar todo tipo de trabajos auxiliares, de acuerdo a requerimientos inmediatos, que no corresponden con su grado de formación profesional. Estas trabajadoras son contratadas con salarios bajos, con un régimen de trabajo caracterizado por la flexibilización funcional, que intensifica y alarga sus jornadas de trabajo (entrevista a un abogado laboralista). En este caso, vemos con claridad, el déclassement asociado a la precariedad laboral. Entre los empleadores del sector financiero, encontramos, también, la práctica de contratar jóvenes diplomados en ciencias económicas y administrativas, como encargados de caja.

La habilidad profesional, los conocimientos técnicos, la inteligencia profesional, el aprendizaje individual, durante varios años, en todos los casos anotados, no parecen preservar al trabajador del déclassement. Esta práctica se la encuentra en distintas regiones, en diferentes sectores económicos y aunque tiene una fuerte presencia entre los diplomados a nivel superior en países de capitalismo desarrollado, está también presente entre de los jóvenes y modestos 
trabajadores de los países latinoamericanos. ¿Cómo explicar la extensión de este desfase entre formación y empleo, que supone la práctica del deéclassement, en los mercados de trabajo? La referencia empírica nos aleja de una explicación de la cualificación, que se base en el reconocimiento de la formación individual del trabajador, más bien, parece negar la existencia de una relación directa entre formación y empleo, propia de una concepción no mercantil del vínculo entre el trabajador y su trabajo, como sostiene G. Friedman(GARCÍA LÓPEZ, $2006)^{11}$.

Considerando la heterogeneidad de las formas y situaciones de déclassement, su explicación parece requerir, la búsqueda de un elemento común a toda formación profesional, en lugar de concentrarnos en las destrezas alcanzadas por los individuos, que siempre nos remiten a casos particulares. El tiempo socialmente necesario, que requiere una determinada formación, aparece como el elemento común, que nos permite, de una parte, explicar el déclassement como un grado de cualificación del trabajo, que no reconoce todo el tiempo de trabajo necesario, para producir una determinada formación profesional; es decir, que la incongruencia entre formación y empleo, nos estaría mostrando que no siempre, todos los contenidos de una formación dada son cualificados y que de las habilidades adquiridas, solo una parte servirá para cualificar, por lo tanto, para clasificar y jerarquizar las actuaciones de uno individuos, respecto a otros en los espacios productivos (Naville, 1979) ${ }^{12}$.

De otra parte, el tiempo socialmente necesario, como elemento común requerido para todo tipo de formación, nos permite salir de la empresa y/o de la fábrica, como espacio exclusivo en el que se produce la relación formación-empleo, para situarnos en un espacio más basto, el del mercado de trabajo. Esto explicaría, por qué individuos con un mismo grado de formación son cualificados en distinto grado, remitiéndonos a la concepción de P. Naville (1979) sobre la cualificación, según la cual, la cualificación del trabajo no puede ser encerrada, ni en el puesto de trabajo, ni en la empresa, ni en la formación del título educativo, sino que se extiende hasta el movimiento ligado con las relaciones reciprocas, que distribuyen las capacidades individuales, entre las diferentes posiciones y modifican sin cesar, sus posiciones

\footnotetext{
${ }^{11}$ Esta Concepción tuvo mucha influencia en el desarrollo, no solo de la investigación académica, sino también de la forma en la que se encaran las políticas educativas en países como Francia. El CEREQ (una institución al servicio de los actores relacionados con la formación y el empleo en Francia) concentró la búsqueda de la adecuación de la formación al empleo, para dar cuenta de la jerarquía del trabajo, considerando entonces, que la tarea consiste en la acción directa sobre la oferta de formación (Tanguy, 2008).

${ }^{12}$ En este sentido, nos precisa García López (2006), que el tiempo de formación con los que el sociólogo organiza las clasificaciones de los trabajadores, expresan en un momento determinado, la relación entre la duración del aprendizaje real y la duración reconocida a través del salario, lo que no significa que exista una relación inmediata entre las remuneraciones económicas, el reconocimiento en los puestos de trabajo a la capacidad de trabajo cualificada y sus reales costes de formación.
} 
y contenidos en los mercados de trabajo(GARCÍA LÓPEZ, 2006), por lo que la evolución de la cualificación no se traduce en la "cualidad "del trabajo que realiza la fuerza de trabajo, sino que se descubre en la escasez social relativa de unas y otras capacidades de trabajo.

El déclassement resultaría de la distancia, entre el conjunto de competencias adquiridas y movilizadas y el conjunto de las competencias efectivamente reconocidas en las empresas como "cualificaciones", las mismas que se definen desde la dinámica de los mercados de trabajo y de la correlación de fuerzas entre los involucrados (patrones y trabajadores). Es decir que el trabajo cualificado, también, se encuentra en permanente movimiento, así como las relaciones, entre los diferentes niveles de trabajo cualificado, porque toda característica de la fuerza de trabajo que se obtenga mediante una educación común, empezará a formar parte del trabajo no cualificado, de lo que se deriva que la duración del aprendizaje, característica de una determinada fuerza de trabajo, no garantiza por siempre, un puesto adecuado a esta duración del aprendizaje y /o un salario fijo de una vez y para siempre (GARCÍA LÓPEZ, 2006).

Esta perspectiva, explicaría, de una parte, que la masificación de la educación superior en Europa, haya producido una sobreoferta de diplomas, que se fueron desvalorizando en el mercado de trabajo, acentuando las posibilidades de déclassement de los jóvenes diplomados o el caso de las empresas chinas, que practican el déclassement en Bolivia, porque sacan ventaja de la presencia de una sobrepoblación relativa, migrantes del campo permanentes, que buscan empleo en el sector de la construcción; pero, también de una creciente sobrepoblación relativa latente (Marx, 1986); o sea, una población de trabajadores agrícolas que, debido al minifundio y la pobreza en el campo, se encuentran en un estado de tránsito permanente al proletariado, dispuestos, cuando las condiciones son favorables, a migrar a las ciudades, para sumarse al proletariado industrial (Castro, 2018). Es importante precisar, que en los distintos casos de déclassement considerados (diplomados supriores, trabajadores de empresas chinas y/o abogadas en empresas estatales, etc.), los patrones pueden hacer uso de mayores competencias de sus trabajadores en los puestos de trabajo, sin que exista un reconocimiento de este trabajo concreto.

La propuesta teórica y epistemología de P. Naville sobre las cualificaciones, nos aproxima: además, a una explicación de la "introuvable" (la no encontrada) relación entre formación y empleo (Tanguy,2008), debido a una perspectiva histórica, que nos sitúa fuera de los límites de una concepción de la cualificación basada en el análisis de trabajo concreto, desconectado del conjunto de la estructura social, para mostrarnos; más bien, el carácter social de la cualificación, en continuo movimiento y transformación, que deshecha toda posibilidad 
de "adecuación ” inmediata, entre formación y empleo. Dinámica que muestra que la relación entre formación y empleo, se produce en términos de transferencias de fuerza de trabajo o de transmutaciones de capacidades y habilidades cognoscitivas, de cambios en el tiempo, dentro de la estructura social. Movimiento que permite comprender, que es, paradójicamente, la separación entre la formación y el empleo, la que, en los mercados de trabajo, abre la posibilidad, para que los empleadores encuentren, en todo momento, los trabajadores que mejor se ajusten a sus requerimientos (García López, 2006), en un mercado en el que la fuerza de trabajo, como capacidad laboral, circula como mercancía (valor de uso y valor de cambio), espacio en el que se homologa, compara y mide, en sus diferencias cualitativas y cuantitativas, en relación, no solo a las diversos procesos de formación de las capacidades laborales (oferta); sino también, a los mecanismos que dan lugar a los espacios de su aplicación productiva; es decir, todos los procesos técnicos y tecnológicos que en la empresa, dan forma a una serie de puestos de trabajo específicos (demanda) (GARCÍA LÓPEZ, 2006).

Esto quiere decir, que una concepción de la cualificación, basada en la habilidad profesional, en los conocimientos profesionales o técnicos, en la inteligencia profesional o el aprendizaje individual, durante varios años, como la propuesta de G. Friedman ¿ino tendría ningún valor explicativo en el funcionamiento de los mercados de trabajo? Si nos remitimos a las investigaciones desarrolladas, al respecto, la respuesta es negativa, las habilidades y conocimientos profesionales, si bien tienen un menor alcance explicativo, acerca de la formación de la cualificación en los mercados de trabajo, que las dimensiones postuladas por P: Naville, la propuesta de G. Friedman nos permite explicar; por ejemplo, por qué los jóvenes con diplomas de nivel superior, en los países europeos son los menos proclives a enfrentar el paro, en relación a jóvenes que no cuentan con estos diplomas, pero esta relación, entre mayor tiempo de formación profesional y menor posibilidad de quedar en paro, no es una relación que se explique en sí misma, pues su comportamiento como relación, esta mediado por el movimiento de un espacio más basto, el del mercado de trabajo.

\subsection{La medicación de las jerarquizaciones sociales en la posibilidad del déclassement}

Numerosas investigaciones concluyen que la posición en el empleo, para un mismo conjunto profesional, varía de acuerdo al sexo, a la edad, a las regiones geográficas, al origen étnico, etc. (IVIE, 2009; GIRET et al. 2006). Es decir, que el déclassement no se presenta de 
igual forma en todos los grupos sociales, es más común en los grupos de trabajadores considerados más vulnerables, como los jóvenes, las mujeres, los migrantes (LINSLEY, 2005), Estudios realizados entre 2012 y 2014 (SHERSHNEVA Y FERNÁNDEZ, 2018), muestran que el fenómeno del déclassement está muy extendido entre la población extranjera, entre la población más afectada, se encuentra la latinoamericana y la proveniente de Europa del Este.

En la población migrante se identificó, que son las mujeres las más expuestas al déclasement profesional. En España, un estudio sobre las mujeres que migran a este país, con fines laborales, identificó que para 2018, el 54,9\% de mujeres estaban en esta situación laboral, frente a un 37\% de los varones (SHERSHNEVA Y FERNÁNDEZ, 2018). Una explicación, atribuye esta situación a que las mujeres se insertan en las actividades menos cualificantes, como el servicio doméstico, la hostelería y el comercio, pero; también, se atribuye esta posición laboral de los migrantes en general y de las mujeres en particular, a la situación administrativa irregular, en que se encuentra esta población y a la falta de convalidación de sus diplomas (adquiridos en el país de origen), en los países europeos, como España. Aunque los mismos estudios precisan, que, si bien un título homologado no garantiza el éxito laboral, a la hora de buscar un empleo, juega un rol importante, por las valoraciones que los empleadores dan al título reconocido por el sistema educativo europeo.

¿Desde una óptica centrada en la relación directa o “natural” entre formación y empleo, cómo explicar que sea la condición de ser mujer, de ser migrante o ser mujer migrante, la que define la posición laboral frente al déclassement, y no el grado de formación? Es decir, la referencia empírica muestra que la posición en los empleos, tiene menos que ver con las competencias movilizadas en los puestos de trabajo, que con jerarquizaciones sociales más amplias, como los estatutos económicos y sociales (NAVILLE, 1956).

Parece que la cualificación no designa; únicamente, una clasificación de puestos de trabajo; sino también, una jerarquía social de funciones, jerarquía que no puede ser comprendida, si no es referida a una jerarquía más amplia, la de los estatutos económicos y sociales (jerarquías que expresan valoraciones sociales, expresadas en ingresos, consumo, etc.). Desde esta perspectiva, es posible precisar, que la relación formación-empleo esta mediada, también, por la división social del trabajo por sexos, que debido a las valoraciones sociales de carácter patriarcal que la permean, nos remite a una desvalorización social del trabajo femenino, en mayor grado que del trabajo de los varones, aunque las mujeres detenten el mismo nivel de formación profesional, esto explicaría, que ellas estén más expuestas al déclassement que los varones. 
Cuando se trata de los trabajadores migrantes, la relación formación-empleo está mediada por la valoración social diferenciada del trabajo, que los ubica, dentro de la jerarquía social, en peldaños inferiores al que ocupan los trabajadores nacionales. No en vano, la discriminación permeada de valoraciones sociales (estereotipos, prejuicios, etc.) es uno de los aspectos más significativos, identificado en estudios dedicados a los mercados de trabajo femeninos y/o de población migrante (SHERSHNEVA Y FERNÁNDEZ ARAGÓN, 2018). Esta población es, también, la más expuesta al déclassement, debido a la presión económica que soporta, para enviar remesas a la familia en su lugar de origen, lo que la lleva a aceptar trabajos, que están por debajo de su nivel de formación.

Se trata de otra ilustración, de que no existe, ningún medio objetivo de cualificar un conjunto de puestos por razones puramente técnicas y que la cualificación es; sobre todo, una apreciación social del valor diferencial de los trabajos (NAVILLE, 1963), un proceso histórico en continua transformación, una relación reconstruida de forma permanente.

\section{Consideraciones finales}

En el transcurso de nuestro artículo, nos propusimos valorar el alcance explicativo que acerca del déclassement, tienen las propuestas teóricas sobre la cualificación en George Friedman y Pierre Naville. El resultado de esta tarea, nos invita a recuperar una de las dimensiones epistemológicas, que da mayor alcance explicativo a la propuesta de P. Naville, la de totalidad, que en este autor, juega un papel explicativo importante, cuando nos señala que el taller y sus situaciones de trabajo, no son sino un momento en una actividad productiva global, que se extiende a diversas cadenas de empresas y, poco a poco, implica a ramas industriales enteras; de donde, el hecho técnico no provoca, directamente, efectos sociales, sino en el seno de determinadas relaciones sociales y económicas, históricamente específicas. Esto quiere decir, que un hecho alcanza su verdadero significado, dentro de la totalidad histórica en la que se produce (KOSIC, 1990). En este sentido, no es que las habilidades profesionales, los conocimientos profesionales o técnicos, la inteligencia profesional o el aprendizaje individual, durante varios años, considerados por G. Friedman, no tengan ningún papel, dentro del proceso de cualificación, lo tienen, como pudimos observar, a la luz de la referencia empírica, pero dentro de procesos sociales más bastos, como la dinámica de los mercados de trabajo o la correlación de fuerzas entre trabajadores y patrones, o las valoraciones sociales, que atribuyen 
jerarquías diversas a los distintos trabajos. Son estos comportamientos, los que terminan por subordinar el rol que tiene la formación en el proceso de cualificación y no al revés.

La otra dimensión epistemológica, que da a la teoría de P. Naville, un mayor alcance explicativo, es el carácter histórico de su propuesta, que atribuye a la cualificación, un comportamiento dinámico, dentro del cual, no es posible una adecuación total de la formación al empleo; más bien, los desajustes entre los estatutos profesionales ofertados por el sistema educativo y los requerimientos del aparato productivo, son considerados por P. Naville un rasgo estructural, de la dinámica que regula la relación formación-empleo (GARCÍA LÓPEZ, 2006), la del mercado de trabajo capitalista, en el que la fuerza de trabajo circula como mercancía y no solo como un valor de uso, como la concibe G. Friedman

La mirada epistemológica propuesta por P. Naville (del mismo modo que la de Friedman) tiene implicaciones importantes para las prácticas formativas, porque plantea la necesidad de que los procesos educativos respondan a la transformación permanente de los procesos productivos. No es casual, por cierto, que el sistema educativo no se dedique a una formación que habilite a las personas, para ocupar un puesto fijo, sino que, más bien, las dota de cierta polivalencia, para que se adapten a varios puestos, frente a las transformaciones tecnológicas y organizativas. Se trata de una socialización de saberes, que finalmente, garantiza, que, en los mercados de trabajo, no falte la fuerza de trabajo requerida, hecho que niega la tesis "adecuasionista", que aunque hacía tiempo que fue abandonada, parece querer, paradójicamente, retornar, pese a que en los mercados de trabajo, la demanda, parece inclinarse por una fuerza de trabajo lo suficientemente polivalente, como para poder adecuarse a los requerimientos productivos de la creciente automatización (Plassard y Thi Thanh Nhu Tran, 2009).

\section{Referências}

LAURENCE, L. Déclassement des jeunes et politiques de l'emploi. Exploration de l'enquête "Génération 98" du CEREQ. Cahiers de la Maison de Sciences Economiques 2005.17, Paris. 2005.

LEMISTRE, P. Déclassement et chômage: Une dégradation pour les plus diplômes. Etat des lieux de 2007 à 2010, en Net.Doc, CEREQ, Paris, May. 2014.

ECKERT, H. Déclassement e hantise du déclassement, en Revue Française de pédagogie : Recherches en éducation, $\mathrm{N}^{\circ}$ 188, ago./sept. 2014. Disponible en $:<\mathrm{http} / /$ journais openeditiom org/rfp/4552 >. Acceso en: 30 sept. 2017. 
MONCEL, N. Quelle qualité d'emploi pour les jeunes diplômés du supérieur, en Formation emploi, $\mathrm{N}^{\circ} 117$, ene./mar. 2012. Disponible en $:<\mathrm{http} / /$ formation emploi.revues.org/3538 >. Acceso en : 5 de jun. 2012.

GIRET, J. Quand les jeunes s'estiment déclassés, dans des formations pour quel emplois? In: Firet J.; Lopez, A.; Rose, J.(eds.), "Recherche "La découverte-CEREQ , p. 279-288. 2005

Buscatto, M. Introduction : "Quand la qualification fait débat”, en formation emploi, Paris, N. 96, p.5-10. 2006

FRIEDMAN, G. Problemas humanos del maquinismo industrial, Buenos Aires, Editorial Sudamericana, 1956.

GARCÍA LÓPEZ, J. El trabajo como relación social: Una problematización del modo de construcción del objeto, a partir de la sociología del salariado de Pierre Naville. 2006. 516 f. Tese (Doctorado) - Universidad Complutense, Madrid. 2006.

NAVILLE, P. "Población activa y teoría de la ocupación”, en Friedman G. y Naville P. (Eds.), Tratado de Sociología del Trabajo, vol. I, Mexico D.F.: Fondo de Cultura Economica, 1978. p. 369-384.

NAVILLE, P. Qu'est-ce-que la qualification du travail ? en L'année sociologique, No 30, pp.497$503,1979$.

GARCÍA, J.; PEIRO, J. y SORO, A. Capital humano: Observatorio de la inserción laboral de los jóvenes: 1996-2002, Bancoja-IVIE, Valencia. 2003.

GARCÍA, J. La inserción laboral de los universitarios y el fenómeno de la sobre cualificación en España, en Papeles de la Economía Española. No 119, p.172-187. 2009.

DOLTON, P. y Bignales, A. the incidence and effects of overeducation in the U.K. graduate labour market. Economic of education review, No 19, p.179-198. 2000.

PASCAL G.; GILLES L. y VINCENT T. Les femmes de Chambre de l'hôtellerie de luxe ou le déclassement d'une élite invisible”, en Revue Formation Emploi, p. 27-44, jul./sep. 2013.

STROOBANTS, M. Savoir-faire et compétences au travail. Une sociologie de la formation des aptitudes. Brucelas, Editions de l'université de Bruxelles, 1993.

MARX, K. El capital, Tomo I, México, Siglo XXI, 1986.

KOSIC, K. La dialéctica de lo concreto, Grijalbo, 1990.

CASTRO, L. Sobreexplotación al estilo chino y su impacto sobre el trabajo en el sector de la construcción. Grupo de estudios del trabajo LLank'aymanta, Cochabamba, jul. 2018.

Disponibleen: <https://llankaymantabolivia.blogspot.com/2018/07/sobreexplotacion-alestilo-chino-y-su_16.html >. Acceso en: 16 jul. 2018.

PLASSARD y THI Thanh Nhu Tran, L'analyse de la sur éducation ou du déclassement: l'escroquerie scolaire enfin démasquée ou beaucoup de bruits pour rien? Celui qui augmente 
Revista Educação e Políticas em Debate - v. 7, n.1, p. 191 - 207 - jan./abr. 2018 - ISSN 2238-8346

sa connaissance augmente sa douleur (Ecclésiaste 18) Il en sait bien trop pour ce qu'il a à faire (Proverbe populaire), en Revue d'Economie Politique, vol.119, p.751-793. 2009.

VINCENT, J. Critique du travail : le faire et l'agir, Paris, PUF, 1987. 